Technical Note

\title{
Design of a dedicated circular coil for Magnetic Resonance Spectroscopy studies in small phantoms and animal acquisition with a 3 Tesla Magnetic Resonance clinical scanner
}

\author{
Giulio GIOVANNETTI ${ }^{\mathrm{a}, \mathrm{b}}$, Alessandra FlORI ${ }^{\mathrm{b}, \mathrm{c}}$, Daniele DE MARCH ${ }^{\mathrm{b}}$, Domenico MONTANARO ${ }^{\mathrm{b}}$, Francesca FRIJIA ${ }^{\mathrm{b}}$ \\ ${ }^{a}$ Institute of Clinical Physiology, National Research Council (CNR), Pisa, Italy \\ ${ }^{b}$ Fondazione CNR/Regione Toscana G. Monasterio, Pisa, Italy \\ ${ }^{c}$ Institute of Life Sciences, Scuola Superiore Sant'Anna, Pisa, Italy \\ *E-mail address: giovannetti@ifc.cnr.it
}

\begin{abstract}
Introduction: Magnetic Resonance Spectroscopy (MRS) is a very powerful tool to explore the tissue components, by allowing a selective identification of molecules and molecular distribution mapping. Due to intrinsic Signal-to-Noise Ratio limitations (SNR), MRS in small phantoms and animals with a clinical scanner requires the design and development of dedicated radiofrequency (RF) coils, a task of fundamental importance. In this article, the authors describe the simulation, design, and application of a ${ }^{1} \mathrm{H}$ transmit/receive circular coil suitable for MRS studies in small phantoms and small animal models with a clinical 3T scanner. In particular, the circular coil could be an improvement in animal experiments for tumor studies in which the lesions are localized in specific areas.

Material and methods: The magnetic field pattern was calculated using the Biot-Savart law and the inductance was evaluated with analytical calculations. Finally, the coil sensitivity was measured with the perturbing sphere method. Successively, a prototype of the coil was built and tested on the workbench and by the acquisition of MRS data.

Results: In this work, we demonstrate the design trade-offs for successfully developing a dedicated coil for MRS experiments in small phantoms and animals with a clinical scanner. The coil designed in the study offers the potential for obtaining MRS data with a high SNR and good spectral resolution.

Conclusions: The paper provides details of the design, modelling, and construction of a dedicated circular coil, which represents a low cost and easy to build answer for MRS experiments in small samples with a clinical scanner.
\end{abstract}

Key words: magnetic field; inductance; signal-to-noise ratio; Magnetic Resonance Spectroscopy; radiofrequency coils.

\section{Introduction}

Magnetic Resonance Spectroscopy (MRS) allows selective identification of molecules and molecular distribution mapping within various organs, and it can be considered a powerful tool to explore the tissue components. In vivo MRS is increasingly applied in the routine clinic for medical imaging. Although the high number of nuclei in the body can provide precious metabolic and physiologic data, such as ${ }^{1} \mathrm{H},{ }^{31} \mathrm{P},{ }^{13} \mathrm{C},{ }^{19} \mathrm{~F}$, and ${ }^{23} \mathrm{Na}$, typically ${ }^{1} \mathrm{H}-\mathrm{MRS}$ is the most used technique thanks to its high sensitivity and the great abundance of hydrogen in a large number of metabolites. ${ }^{1}$ Nowadays, ${ }^{1} \mathrm{H}-\mathrm{MRS}$ of the brain is a leading application in the clinical setting, providing information on different key molecules, in several pathologic conditions. $^{2}$ However, MRS of small samples (such as phantoms or animal models) would require the use of very high field scanners (7T or above), which allows enhancing the Signal-to-Noise Ratio (SNR) thanks to the linear dependence of the Magnetic Resonance (MR) signal on the $B_{0}$ static magnetic field intensity. ${ }^{3}$ Nevertheless, few laboratories and clinical research groups have easy access to such dedicated MRS systems and a cheap solution could be to perform MRS by using conventional clinical scanners, typically with static magnetic field strengths of 1.5-3T. The use of clinical scanners for animal experiments offers some advantages, such as the possibility to use acquisition protocols optimized for the clinic and to approach tissue contrast typical of human studies, because the $\mathrm{T} 1$ and $\mathrm{T} 2$ relaxation times change at high-field strength strongly influence the contrast effect. ${ }^{4}$ Moreover, radiofrequency (RF) power deposition and tissue heating are significantly lower with clinical scanners, leading to low Specific Absorption Rate (SAR), ${ }^{5}$ which can be further reduced when specific coils are employed, since RF heating is confined to the local anatomic region and not to the entire body or organ.

However, in order to minimize the intrinsic problem of low SNR in clinical scanners, the design and the development of dedicated RF coils are necessary constraints in MRS exams 
with small phantoms. The literature contains a good summary of RF MR coils classification according to their shapes (surface, volume, and phased-array coils) ${ }^{6}$ and a comparison of different miniaturized coil geometries (solenoid, planar helical, and transmission-line type), ${ }^{7}$ where the size of the coil were designed for the analysis of nanoliters sample volumes with dedicated Nuclear Magnetic Resonance (NMR) spectrometers. Another paper describes a probe constituted by eight solenoid coils constructed for high-resolution NMR spectroscopy of different chemical solutions at $14 \mathrm{~T}^{8}$

Regarding RF coil technology for small phantoms and animal models with clinical MR scanners, our group has recently worked on the development of coils with different geometries in dependence on the desired field-of-view (FOV), such as an elliptical loop for elongated regions which matches with the mouse body shape ${ }^{9}$ or a Helmholtz coil whose dimensions are optimized for imaging and spectroscopy in small phantoms, ${ }^{10}$ both with a $3 \mathrm{~T}$ MR clinical scanner. The first paper ${ }^{9}$ reports even a comparison (in terms of magnetic field intensity/distribution and penetration depth) between elliptical, circular, square, and rectangular loops which cover the same sample portion in terms of geometric area, although with different shapes.

Evaluation of image quality and spatial resolution on a clinical whole-body $3 \mathrm{~T}$ scanner using different coils for small animal imaging, including a $3 \mathrm{~cm}$ diameter loop which was placed directly on the rat's head for optimal SNR, was described in literature. ${ }^{11}$ However, details about the design and test of such coil were not provided by the authors.

A more sophisticated coil geometry was described in a paper $^{5}$ which details the design of a four-channel phased array receive-only coil for imaging of small animal central nervous system pathologies with a $3 \mathrm{~T}$ clinical scanner.

Although it is almost never mentioned in the literature, the dedicated RF coils have to be designed for being compatible with commercial scanners, because they have to be connected to the scanner and generally the interconnection is achieved via $50 \Omega$ coaxial cables. Despite the coils have to be matched to this impedance value for optimizing the energy transfer through all parts of the spectrometer, different MR scanners could use various connectors and this issue has to be taken into account during the coil design and building.

Our question regards the possibility to provide a low cost and easy to build a solution for ${ }^{1} \mathrm{H}$ MRS in small animals examination of brain metabolites and in extracts of biological sample by using a clinical MR scanner at 3T.

However, due to the smaller region of interest (ROI) and low metabolite concentration with respect to Magnetic Resonance Imaging (MRI), ${ }^{12}$ coil dimensions should be minimized to improve the SNR and spectral resolution, even if at the expense of reduced field homogeneity. The simplest design of a small coil is a circular loop made out of copper, which is able to produce a magnetic field orthogonal to the coil plane in the central ROI whose intensity falls along the coil axis. ${ }^{13}$

In this paper, the simulation and design of a circular coil for MRS studies in small phantoms and animal models to be used in a commercial MR scanner is reported. After the magnetic field pattern estimation, the conductor inductance calculation was performed using the integral method and the home-built coil was tuned at $127.75 \mathrm{MHz}$. Successively, coil sensitivity was measured at the workbench with the perturbing sphere method, ${ }^{14,15}$ which is able to provide, in a fast and easy way, RF coil field mapping and which can be useful for periodical coil quality controls in a short time.

The prototype was designed to be used in transmit/receive mode with a $3 \mathrm{~T}$ clinical scanner by employing a T/R switch inserted between the coil and the scanner and provided by the scanner manufacturer, which permits monitoring the SAR for avoiding harm to the sample. Preliminary ${ }^{1} \mathrm{H}$ MRS phantom studies were carried out to further characterize the coil performance by comparing spectra SNR with the ones provided by a standard commercial head coil, which is commonly employed for MR spectroscopic imaging of the human brain. ${ }^{16}$

\section{Methods}

\section{Magnetic field estimation}

A convenient formulation for calculating the magnetic field from a line current employs the magnetostatic theory, which is applicable to coils whose size is a small fraction $(<1 / 10)$ of the wavelength associated with $\mathrm{B}_{0}{ }^{17}$ The Biot-Savart law permits to calculate the $\mathrm{B}_{1}$ field components at $\mathrm{P}(\mathrm{x}, \mathrm{y}, \mathrm{z})$ location position of a $b$ radius circular loop carrying an electric current $I$ (Figure 1a) as ${ }^{18}$ :

$$
\begin{aligned}
& B x_{p}=\frac{\mu_{0} I}{4 \pi} \int_{0}^{2 \pi} \frac{b y \cos \theta d \theta}{\left[(x-b \cos \theta)^{2}+y^{2}+(z-b \sin \theta)^{2}\right]^{\frac{3}{2}}} \\
& B y_{p}=\frac{\mu_{0} I}{4 \pi} \int_{0}^{2 \pi} \frac{\left(b^{2}-b z \sin \theta-b x \cos \theta\right) d \theta}{\left[(x-b \cos \theta)^{2}+y^{2}+(z-b \sin \theta)^{2}\right]^{\frac{3}{2}}} \\
& B z_{p}=\frac{\mu_{0} I}{4 \pi} \int_{0}^{2 \pi} \frac{b y \sin \theta d \theta}{\left[(x-b \cos \theta)^{2}+y^{2}+(z-b \sin \theta)^{2}\right]^{\frac{3}{2}}}
\end{aligned}
$$

where $\mu_{0}=4 \pi^{*} 10^{-7}$ henrys per meter $(\mathrm{H} / \mathrm{m})$ is the free space permeability. $\mathrm{P}(\mathrm{x}, \mathrm{y}, \mathrm{z})$ space field point and the position of the points in the loop path are expressed, respectively, in Cartesian and polar coordinates.

\section{Inductance calculation}

The inductance of the circular loop was calculated by schematizing the wire conductor constituting the loop as part of a circular segment (Figure 1b) and section (Figure 1c) and by using the following expression: ${ }^{18}$

$$
\begin{aligned}
& L_{c i r c}=\frac{\mu_{0}}{4 \pi(2 \pi a)^{2}} \int_{0}^{2 \pi} \int_{0}^{2 \pi} \int_{0}^{2 \pi} \int_{0}^{2 \pi} \frac{\cos (\theta t-\theta)}{W} a^{2}(b+a \cos \phi)(b+ \\
& \left.a \cos \phi^{\prime}\right) d \phi^{\prime} d \theta^{\prime} d \phi d \theta
\end{aligned}
$$

where $a$ is the wire conductor radius and $W=\left|r-r^{\prime}\right|$. 


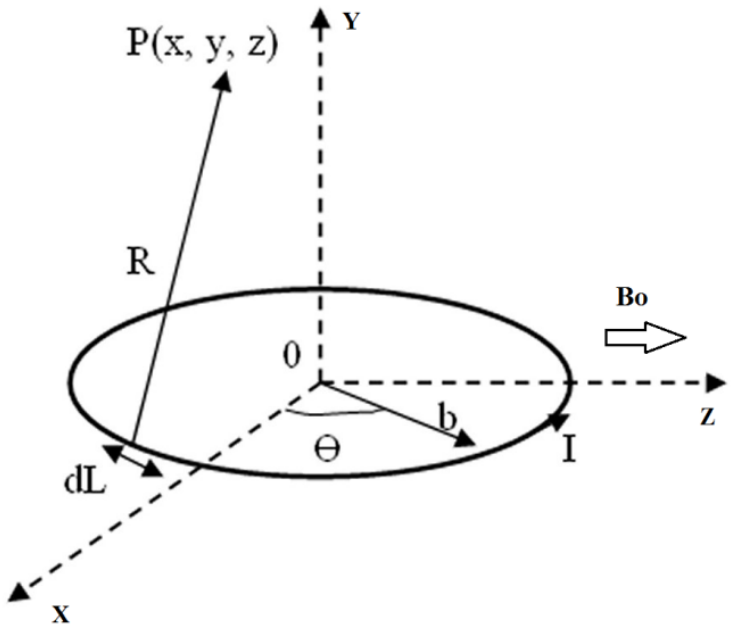

(a)

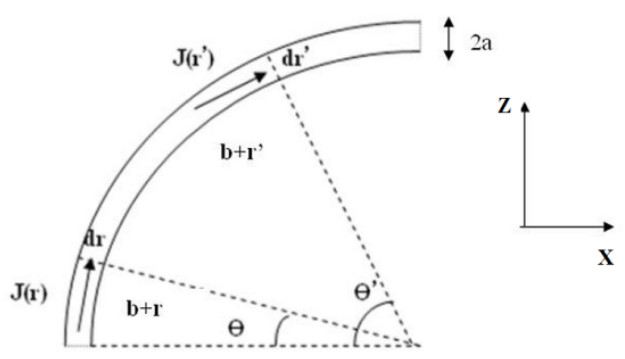

(b)

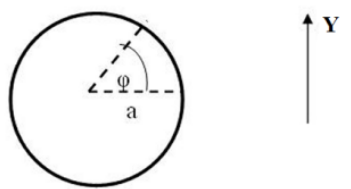

(c)

Figure 1 Circular loop geometry in the reference system used to calculate the magnetic field components given by Equations 1, 2 and 3 (1a) and to calculate the loop inductance (1b: upper view of a coil segment; 1c: section view ot the wire used in the coil)

\section{Coil sensitivity estimation}

Coil sensitivity is a characterizing parameter of the RF coil performance, defined as the magnetic field $\left(B_{l}\right)$ induced by the coil at a given point per unit of supplied power $P$, as follows: ${ }^{19}$

$\eta=\frac{B_{1}}{\sqrt{P}}$

Eq. 5

In this work, the coil sensitivity $\eta$ was measured using the perturbing sphere method, an electromagnetic bench test classified among "probe techniques" which consists in putting a small metallic sphere near the coil and measuring the frequency shift with respect to the unloaded coil. Successively, the following equation has to be used:

$\eta=\frac{B_{1}}{\sqrt{P}}=\frac{1}{2} \sqrt{\left(\frac{\mu_{0}}{\pi^{2} B_{w} r_{S}{ }^{3}}\right)\left(\frac{f_{1}{ }^{2}-f_{0}{ }^{2}}{f_{0}{ }^{2}}\right)}$

where $B_{1}$ is the rotating component of the magnetic field, $B_{w}$ and $f_{0}$ are, respectively, the $-3 \mathrm{~dB}$ bandwidth and the resonant frequency of the unloaded coil, $f_{l}$ is the coil resonant frequency after the sphere insertion and $r_{s}$ is the sphere radius, which should be placed in a region of zero electric field.

Being the electric field negligible in the coil axial direction, the coil magnetic field mapping can be estimated by varying the sphere position, with sensitivity values calculated at different z-coordinates. For such measurements, the network analyzer was set in an averaging mode for improving measurement sensitivity.

\section{Coil design}

The circular loop is a coil configuration used in different applications when it is less important to get a signal from the whole sample than to get as much signal as possible from a small region of interest (ROI). As a rule of thumb, for maximizing the SNR in the ROI, the coil radius should roughly match the depth of the desired imaging. ${ }^{20}$ Since for our application the sample region extends up to $2 \mathrm{~cm}$ in depth with respect to the coil plane, a $4 \mathrm{~cm}$ diameter circular loop was designed by using a $0.2 \mathrm{~cm}$ diameter wire conductor.

Coil matching and tuning at $127.75 \mathrm{MHz}$ were performed using high quality capacitors (ATC 100C - American Technical Ceramics, USA). The coil matching was achieved by using a capacitive circuit which transforms the coil impedance to $50 \Omega$ according to the following expression: ${ }^{21}$

$\frac{1}{2} C_{m}=\sqrt{\frac{C}{2 \pi f_{0} Q 50}}$

where $C$ is the coil tuning capacitor, $Q$ is the coil quality factor in loaded condition and $f_{0}$ is the resonant frequency. Finally, the coil fine-tuning within the MR system was performed by using a variable capacitor $C v$ (AP40HV Voltronics, USA). Figure 2 shows a sketch of the built coil.

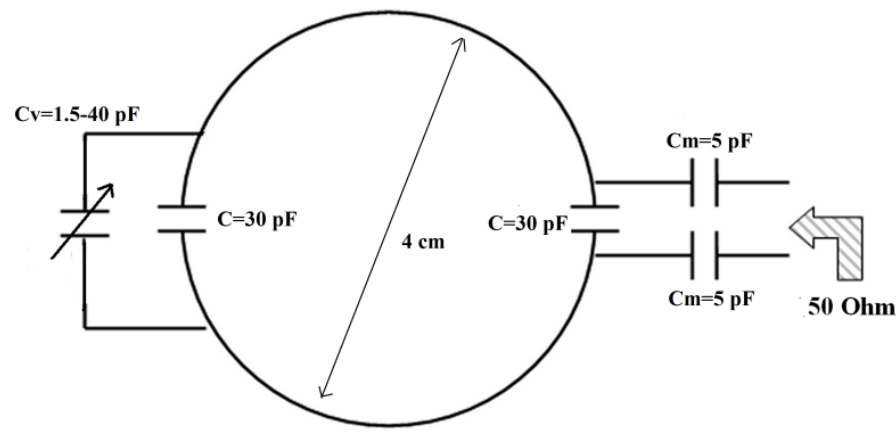

Figure 2. Electric scheme of the loop coil showing the matching network and the tuning capacitors 


\section{Workbench test}

Before the insertion of capacitors, coil inductance $L$ was initially measured at $127.75 \mathrm{MHz}$ with workbench instrumentation consisting of a network analyser HP3577 (Hewlett Packard, Palo Alto, CA) and by connecting the coil to the analyzer with RG58 coaxial cable after performing a proper calibration.

Successively, coil quality factor measurements were performed with the same analyzer and by using a homebuilt dual-loop consisting of two pickup circular loops, which are partially overlapped for minimizing the mutual coupling between the elements: this is achieved by separating the centers of the loops by about 0.75 times their diameter. ${ }^{22}$ The measurements were conducted using one loop coil as a transmitter and the other one as a receiver. With this setup, the transmit loop was weakly coupled to the circular coil, which in turn was weakly coupled to the receive loop. The power transmitted from the transmit to the receive loop resulted proportional to the amplitude of the oscillation in the circular coil and, therefore, represented its frequency response. ${ }^{19}$

The evaluation of the coil quality factor for circuit matching design was performed by using a load constituted by a cylindrical vial $(10 \mathrm{~cm}$ long and $2.5 \mathrm{~cm}$ diameter) filled with deionized water.

Finally, the coil sensitivity was evaluated at the center of the loop and at a y-coordinate of $1.3 \mathrm{~cm}$ along the coil axis, using a metallic perturbing sphere of $1.2 \mathrm{~cm}$ diameter.

\section{Phantom Magnetic Resonance Spectroscopy test}

To evaluate the efficiency of the designed coil, spectroscopic profiles were acquired with a 3T GE Excite HDx (GE Healthcare, Waukesha, WI) clinical scanner and compared with the ones acquired using a head coil constituted by an 8elements high-resolution brain array. The same spectroscopic sequence and the same phantoms (three metabolic compounds part of the brain) were used for the comparison.

To test the coil we prepared the same small phantoms that are used for understanding the metabolic pathway in humans. The first phantom used in this experiment consisted of two 500 $\mu \mathrm{L}$ centrifuge tubes containing respectively a $250 \mathrm{mM}$ solution of Myo-inositol (Myo) and a $100 \mathrm{mM}$ solution of choline chloride (Cho), both in purified $\mathrm{H}_{2} \mathrm{O}$, and fixed to the cap of a $50 \mathrm{~mL}$ conical centrifuge tubes filled with purified water. Each container was filled to minimize the presence of air bubbles.

The second phantom consisted of $150 \mu \mathrm{L}$ of $1 \mathrm{M}$ Sodium-Llactate (Lac) in $\mathrm{H}_{2} \mathrm{O}$. The solution was placed in a small plastic container maintained at the middle height of a $50 \mathrm{~mL}$ conical centrifuge tube by a plastic holder fixed at one end to the cap of the tube. The $50 \mathrm{~mL}$ conical centrifuge tube was filled with purified water and the small plastic container was sealed with a plastic paraffin film.

The phantoms were then positioned with their axes perpendicular to the coil plane starting from $y=0$ coordinate, in order to place the $500 \mu \mathrm{L}$ centrifuge tubes in the maximum strength region of the $\mathrm{B}_{1}$ field.

Reference ${ }^{1} \mathrm{H}$ images of the phantom were obtained with the body coil by using a fast spin-echo (FSE) sequence (FOV $=80$ x $80 \mathrm{~mm}, 512$ x 512 matrix, slice thickness $=2.5 \mathrm{~mm}, \mathrm{TE} / \mathrm{TR}=$ $43.2 \mathrm{~ms} / 2740 \mathrm{~ms}$, number of averages $=4$ ).

Voxel for localized Point RESolved Spectroscopy (PRESS) spectra was located in the center of the phantom. The core sequence consists of three slice-selective RF-pulses $\left(90^{\circ}-180^{\circ}-180^{\circ}\right)$ applied concurrently with three orthogonal gradients ( $\mathrm{x}, \mathrm{y}$, and $\mathrm{z}$ ). Outer-volume and CHESS water suppression techniques were incorporated in the PRESS sequence. TE was chosen to be $35 \mathrm{~ms},{ }^{23} \mathrm{TR}=2 \mathrm{sec}$, voxel volume $20 \times 20 \times 20 \mathrm{~mm}$, spectral resolution $=2048 \mathrm{pts}$, bandwidth $=5000 \mathrm{~Hz}$, the total number of scan $=512$, given a total acquisition time of $17.52 \mathrm{~min}$.

All spectra were reconstructed with SAGE software package (version 7.7, GE Healthcare) using a dedicated semi-automated program (PROBE/SVQ). ${ }^{24}$

Residual water was removed in the time domain. The timedomain FID signals were then Fourier transformed to yield the MR spectra, which were phase and baseline corrected. The generated spectra were then fitted to Lorentzian line shapes using the Levenberg-Marquardt method of nonlinear leastsquares minimization.

Spectral quantification was performed by manually selecting (in the frequency domain) the detected resonances (Myo, Cho, and Lac).

A table of the estimated peak amplitude, linewidth, and frequency was then automatically generated.

To compare the quality of the spectra acquired with the standard head coil and the circular coil we evaluated SNR as:

$S N R=\frac{S_{j}}{\sigma_{\text {noise }}}$

where the amplitude of each metabolite $S j$ was evaluated in the voxel while the noise level was calculated in the same voxel as the standard deviation $\sigma_{\text {noise }}$ of the remaining data points in the signal-free region ( 0 to $-1 \mathrm{ppm}){ }^{25,26}$

Furthermore, the full width at half maximum (FWHM) of each metabolite was measured for both coils.

\section{Results}

\section{Coil simulation}

Inductance and magnetic field patterns were evaluated by using IDL 6.0 (Interactive Data Language, Visual Information Solutions, Boulder, CO, USA) software tool.

An inductance of $75.52 \mathrm{nH}$ was calculated with Equation 4 for the circular loop.

The diagram in Figure 3 refers to the magnetic field patterns of the circular loop calculated to a y-coordinate of $1 \mathrm{~cm}$, while Figure 4 shows the profile plot (along the y-axis) of the magnetic field pattern in dependence on the depth profile. 


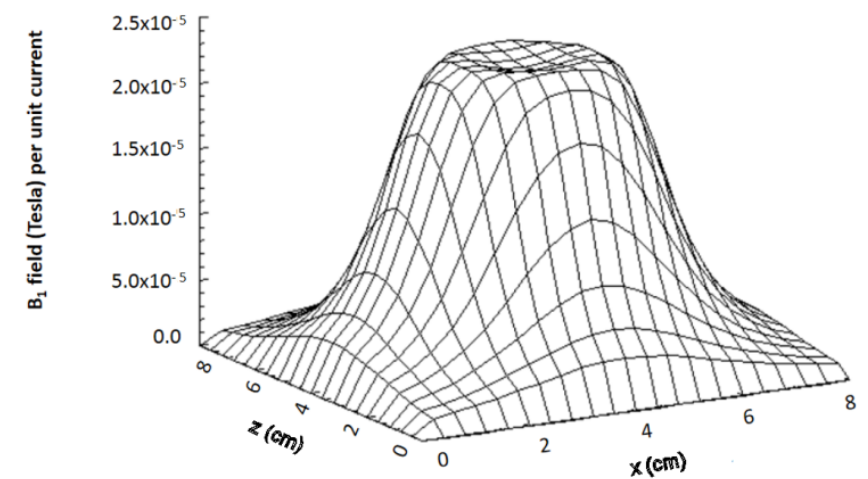

Figure 3. Theoretical normalized 3D magnetic field for the circular coil at $\mathbf{y}=1 \mathrm{~cm}$

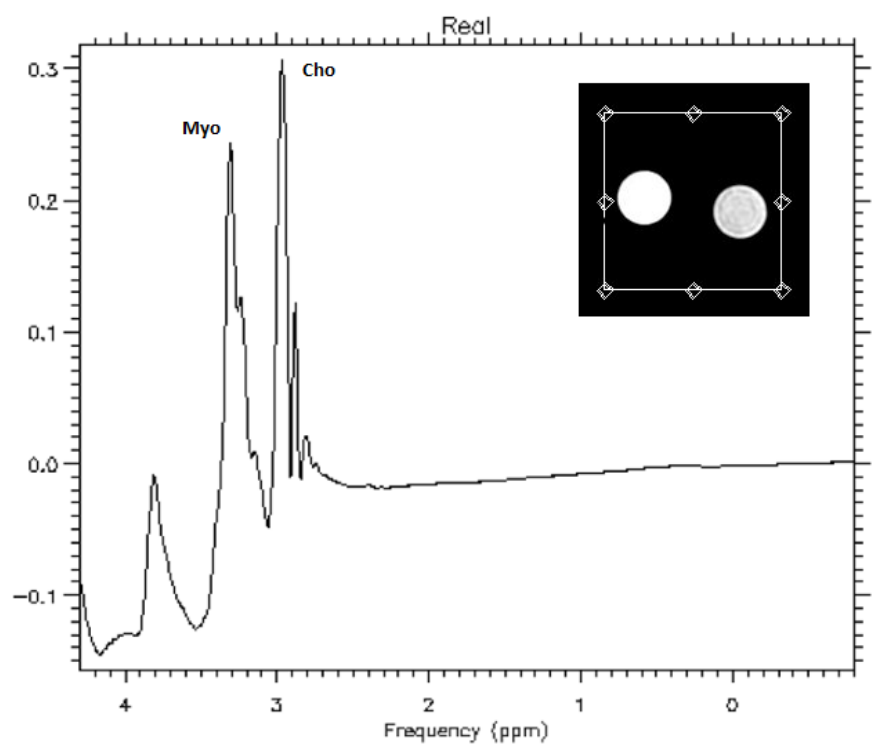

Figure 5. Myo-Inositol and Choline Chloride spectrum obtained at 3T with the circular coil developed in this work, with the localization of the voxel for MRS. The phantoms were positioned with their axes perpendicular to the coil plane starting from $y=0$ coordinate.

\section{Coil workbench test}

The measured inductance value $L$ was $73.78 \mathrm{nH}$. The value of the capacitance $C$ needed for coil tuning resulted to be $30 \mathrm{pF}$, while the fine-tuning was performed by using a $1.5-40 \mathrm{pF}$ variable capacitor $C v$ (Figure 2).

Unloaded quality factor $Q_{U}$ resulted to be 201, while the measurement of the quality factor $Q_{L}$ in loaded condition by using the previously described phantom provided a value of 114 , corresponding to an $r$ value of $1.76\left(r=Q_{U} / Q_{L}\right),{ }^{20}$ which is a common coil performance parameter since $S N R \alpha \sqrt{(1-}$ $1 / r) .^{27}$

The measurement of loaded quality factor permitted to characterize the receiver system $T_{d}$ dead time at the resonant frequency $\left(T_{d}=30 Q_{L} / 2 \pi f_{0}\right)$, which resulted to be $4.3 \mu \mathrm{s}^{28}$

Subsequently, a coil matching of $-20 \mathrm{~dB}$ at $127.75 \mathrm{MHz}$ was achieved by input port reflection coefficient (S11) measurements in the same loaded condition, by using $5 \mathrm{pF}$ capacitors $C_{m}$ calculated using Equation 7 (Figure 2).

Table 1 reports the obtained sensitivity values as the mean value \pm standard deviation.

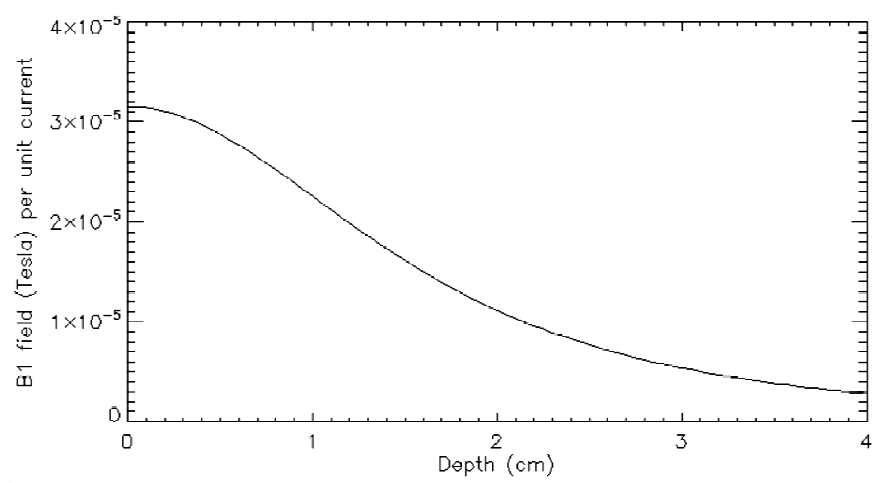

Figure 4. Theoretical normalized magnetic field in the $y$-axis vs depth profile for the circular coil

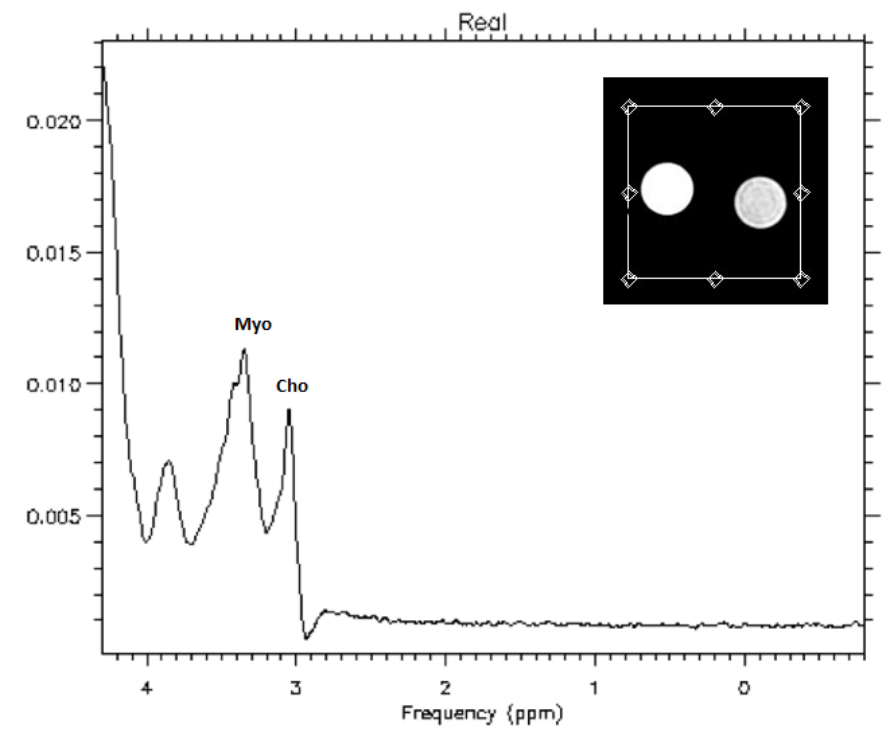

Figure 6. Myo-Inositol and Choline Chloride spectrum obtained at 3T with the commercial coil, with the localization of the voxel for MRS

The accuracy of the perturbing sphere method can be evaluated by calculating the ratio between the coil sensitivity values obtained at the two different $y$-coordinates (sensitivity at $\mathrm{y}=0$ $\mathrm{cm} /$ sensitivity at $\mathrm{y}=1.3 \mathrm{~cm}$ ) and provided a value of 1.68 . This is very similar to the ratio between the magnetic field per unit current (see Figure 4) calculated at $y=0 \mathrm{~cm}(0.315 \mathrm{G} / \mathrm{A})$ and at $y=1.3 \mathrm{~cm}(0.186 \mathrm{G} / \mathrm{A})$, which resulted to be equal to 1.69 .

\section{Phantom Magnetic Resonance Spectroscopy test}

Preliminary results for the MRS acquisitions with the circular coil and the Myo - Cho phantom are shown in Figure 5. The two peaks attributed to Myo-Inositol and Choline Chloride, respectively, are located at $3.3 \mathrm{ppm}$ and $2.97 \mathrm{ppm}$ and properly spaced, resonating at $0.3 \mathrm{ppm}$ apart from each other.

To prove the quality of spectra in our study obtained with the circular coil for small phantom we compared the profile of the spectra using the head coil with the same sequence, phantom, and spatial resolution as depicted in Figure 6. 
Moreover, Table 2 shows the comparison of the SNR values calculated as described in Equation 8 on Myo-inositol peak which was positioned in the coil axis at a distance of $1.3 \mathrm{~cm}$ with respect to the coil plane.

The SNR was also evaluated for the lactate phantom, for spectra acquired at a distance of $y=0 \mathrm{~cm}$ and $\mathrm{y}=1.3 \mathrm{~cm}$ and the results are summarized in Table 3.

Spectral data from both phantoms demonstrated that the SNR in the circular coil was higher than that in the head coil.

Spectral resolution is accurately defined in terms of the full width at half maximum (FWHM): the results for the planar coil $(\mathrm{FWHM}=8 \mathrm{~Hz}$ ) and for the head coil (FWHM=10 Hz), provide reasonable values for a $3 \mathrm{~T}$ scanner and can be compared among them.

\section{Discussion}

In this work, details about the design, simulation, and test of a circular coil to be used in transmit/receive mode for MRS studies with a clinical $3 \mathrm{~T}$ scanner are reported. The proposed coil provided a device with optimized dimensions for spectroscopy in small phantoms and animals for studies of localized areas, to be integrated with a $3 \mathrm{~T}$ clinical MR scanner.

In particular, the simulation permitted us to estimate inductance, magnetic field pattern, and sensitivity of the coil. Successively, a coil prototype was tested on the laboratory workbench for the assessment of the parameters. A difference of only $2.4 \%$ was found between the predicted $L$ value and the experimental value.

The perturbing sphere method allowed the coil sensitivity estimation, whose accuracy was verified by comparison with the magnetic field profile obtained with coil simulations.

Focusing on the applications, the coil was initially employed for the acquisition of MR ${ }^{1} \mathrm{H}$ spectra, showing its capability to discriminate adjacent peaks in a spectrum, mainly dependent on spectral distance, i.e., the difference in resonance frequency between two metabolites. The circular coil provided a good spectral resolution reflected by the Myo and Cho peaks separation, in line with the standard commercial head coil.

There is no agreement among experts on what exactly defines a good spectrum. Literature ${ }^{29}$ provided a list of issues important for the judgment of spectral quality and a series of good and bad spectra to demonstrate the artifacts that one should look out for. High-quality spectra are generally characterized by metabolite signals, which are large compared to the noise level and well separated. These spectral quality features can be quantitatively described by the SNR and the FWHM of resonance peaks as a measure of linewidth and/or spectral resolution.

Anyway, in our spectra acquisitions, we excluded hardware problems $\left(\mathrm{B}_{0}\right.$ inhomogeneity, eddy currents, $\mathrm{RF}$ and gradient amplifiers malfunction, various noise sources) at the scanner that can potentially lead to bad spectral quality, artifacts, and misinterpretation of MR spectra.
Table 1. Sensitivity measurements for the circular coil at two different $y$-coordinates. Each efficiency value has been calculated as the average of four measurements \pm a standard deviation

\begin{tabular}{ccc}
\hline \hline Coil sensitivity & $\mathbf{y}=\mathbf{0} \mathbf{~ c m}$ & $\mathbf{y}=\mathbf{1 . 3} \mathbf{~ c m}$ \\
\hline$(\mu T / \sqrt{W})$ & $30.13 \pm 0.44$ & $17.91 \pm 0.63$ \\
\hline \hline
\end{tabular}

Table 2. SNR values of Myo-inositol peak provided by the two different coils

\begin{tabular}{cc}
\hline \hline Coil & Signal-to-Noise Ratio \\
\hline Circular loop at $\mathrm{y}=1.3 \mathrm{~cm}$ & 276 \\
Head coil & 90 \\
\hline \hline
\end{tabular}

Table 3. SNR values of Lactate peak provided by the two different coils

\begin{tabular}{cc}
\hline \hline Coil & Signal-to-Noise Ratio \\
\hline Circular loop at $\mathrm{y}=0 \mathrm{~cm}$ & 1428 \\
Circular loop at $\mathrm{y}=1.3 \mathrm{~cm}$ & 236 \\
Head coil & 87 \\
\hline \hline
\end{tabular}

These reported elements that prove the good quality of the spectra suggested the possibility to use the circular coil with a clinical scanner for small phantom and small animal studies, in particular for tumor localized in a specific area.

Although the spectral resolution in NMR spectroscopy (and MRS of course) depends solely on the homogeneity of the main magnetic field $\mathrm{B}_{0}$, one can take advantage of the nonhomogeneous $B_{1}$ field of the circular coil to reduce the region of interest where the $\mathrm{B}_{0}$ field is homogeneous.

As shown in Figure 4, the proposed coil provided a suitable field homogeneity in the detected region of interest covering the phantom, resulting in a proper spectral resolution for the studied molecules.

Moreover, the circular coil provided an enhanced sensitivity within MRS experiments, as shown by the higher SNR values reported in Tables 2 and 3. This is mainly ascribable to the circular coil dimensions that better fit the small sample size, as compared to the commercial head coil.

The improved SNR obtained at 3T with the designed circular coil could be also used to provide finer spatial resolution with the same acquisition time.

Although a solenoid could be used for favoring the magnetic field homogeneity, the designed circular coil was optimized for achieving the highest local SNR when placed in close contact with the anatomical region: it is a classical configuration employed in many applications when it is less important to get signal from the whole sample than to get as much signal as possible from a small ROI, for example in the mouse brain and cancer studies.

In the paper, only preliminary results were reported and further work is needed to fully characterize the coil performance for MRS, especially for in vivo studies.

In particular, it is well known that the ideal setup should comprise the use of two different coils: a transmit coil, constituted by a homogeneous volume resonator for achieving an efficient excitation in a large volume, and a receive coil, 
constituted by high local sensitivity. Future work will regard adding a passive decoupling circuit to the circular coil for using it as a receive-only coil while transmitting with the whole body coil, in order to use the good field homogeneity of this last during excitation. Moreover, another issue which will be investigated regards how the use of a multiturn conductor affects the circular coil performance in terms of $r$ ratio and SNR, since an SNR gain up to $20 \%$ could be obtained by building a similar coil with two turns ${ }^{30}$.

\section{Conclusion}

This work describes the design of a home-built RF transmit/receive coil intended to be used for ${ }^{1} \mathrm{H}$ spectroscopy in small animals examination of brain metabolites and in an extract of a biological sample using a clinical MR scanner at
3T. The coil design is a circular single loop and the great advantage of this approach is that the design is really cheap, simple to build, and can be precisely fitted to the investigated sample. The results summarized in this paper provide both the electrical properties of the coil and the spatial distribution of the magnetic component of the RF field, which is related to the spatial sensitivity.

In particular, the presented data show that the designed coil offers the potential for obtaining MRS data with a higher SNR and good spectral resolution and suggests a potential further application of the proposed coil, besides small animal MRI. The theoretical approaches described in this paper could be interesting for graduate students and researchers working in the field of MR coil design and development.

\section{References}

1. Faghihi R, Zeinali-Rafsanjani B, Mosleh-Shirazi M-A, et al. Magnetic Resonance Spectroscopy and its Clinical Applications: A Review. J Med Imag Rad Sci 2017;48:233-253. DOI: 10.1016/j.jmir.2017.06.004

2. van der Graaf M. In vivo magnetic resonance spectroscopy: basic methodology and clinical applications. Eur Biophys $\mathbf{J}$ 2010;39:527-540. DOI: 10.1007/s00249-009-0517-y

3. Hoult DI, Richards RE. The signal-to-noise ratio of the nuclear magnetic resonance experiment. J Magn Reson 1976;24:71-85. DOI: 10.1016/j.jmr.2011.09.018

4. Herrmann K-H, Schmidt S, Kretz A, et al. Possibilities and limitations for high resolution small animal MRI on a clinical wholebody 3T scanner. Magn Reson Mater Phy 2012; 25(3):233-244. DOI: 10.1007/s10334-011-0284-5

5. Pillai DR, Heidemann RM, Kumar P, et al. Comprehensive small animal imaging strategies on a clinical 3 T dedicated head MRscanner; adapted methods and sequence protocols in CNS pathologies. PLoS ONE 2011;6(2):e16091. DOI: 10.1371/journal.pone.0016091

6. Giovannetti G, Hartwig V, Positano V, Vanello N. Radiofrequency coils for Magnetic Resonance applications: theory, design and evaluation. Critical Reviews in Biomedical Engineering 2014;42(2):109-135. DOI: 10.1615/critrevbiomedeng.2014011482

7. Fratila RM, Velders AH. Small-volume Nuclear Magnetic Resonance Spectroscopy. Annu Rev Anal Chem 2011;4:227-249. DOI: 10.1146/annurev-anchem-061010-114024

8. Wang H, Ciobanu L, Edison AS, Webb AG. An eight-coil high-frequency probehead design for high-throughput nuclear magnetic resonance spectroscopy. J Magn Reson 2004;170(2):206-212. DOI: 10.1016/j.jmr.2004.07.001

9. Giovannetti G, Flori A, De Marchi D, et al. Simulation, design and test of an elliptical surface coil for Magnetic Resonance Imaging and Spectroscopy. Conc Magn Reson Part B 2018; 47B(4):e21361. DOI: 10.1002/cmr.b.21361

10. Giovannetti G, Frijia F, Flori A, Montanaro D. Design and simulation of a Helmholtz coil for Magnetic Resonance Imaging and Spectroscopy experiments with a 3T MR clinical scanner. App Magn Reson 2019;50(9):1083-1097. DOI: 10.1007/s00723-01901141-9

11. Herrmann K-H, Schmidt S, Kretz A, et al. Possibilities and limitations for high resolution small animal MRI on a clinical wholebody 3 T scanner. Magn Reson Mater Phy 2012; 25(3):233-244. DOI: 10.1007/s10334-011-0284-5

12. Tomanek B. Radio Frequency Coils for Magnetic Resonance Spectroscopy. In: Webb GA (eds). Modern Magnetic Resonance. Dordrecht: Springer; 2008. p.1163-1170.

13. Mispelter J, Lupu M, Briguet A. NMR probeheads for biophysical and biomedical experiments. London: Imperial College Press; 2015.

14. Maier LC, Slater JC. Field strength measurements in resonant cavities. J App Phys 1952;23:68-77. DOI: 10.1063/1.1701980

15. Giovannetti G, Frijia F, Menichetti L, et al. Coil sensitivity estimation with perturbing sphere method: application to $13 \mathrm{C}$ birdcages. App Magn Res 2012;42:511-518. DOI: 10.1007/s00723-012-0323-z

16. Soher BJ, van Zijl PC, Duyn JH, Barker PB. Quantitative proton MR spectroscopic imaging of the human brain. Magn Reson Med 1996;35: 356-363. DOI: 10.1002/mrm.1910350313

17. Jin J. Electromagnetic Analysis and Design in Magnetic Resonance Imaging. Boca Raton: CRC; 1998. 
18. Giovannetti G. Comparison between circular and square loops for low-frequency magnetic resonance applications: theoretical performance estimation. Conc Magn Reson Part B 2016; 46B:146-155. DOI: 10.1002/cmr.b.21343

19. Darrasse L, Kassab G. Quick measurement of NMR-coil sensitivity with a dual-loop probe. Rev Sci Instrum 1993;64:1841-1844. DOI: $10.1063 / 1.1144020$

20. Haase A, Odoj F, Von Kienlin M, et al. NMR probeheads for in vivo applications. Conc Magn Reson 2000;12 (6):361-388. DOI: 10.1002/1099-0534(2000)12:63.3.CO;2-C

21. Chen C, Hoult DJ. Biomedical magnetic resonance technology. Bristol: IOP;1989.

22. Roemer PB, Edelstein WA, Hayes CE, Souza SP, Mueller OM. The NMR phased array. Magn Reson Med 1990;16:192-225. DOI: 10.1002/mrm. 1910160203

23. Zhu H, Barker PB. MR Spectroscopy and Spectroscopic Imaging of the Brain. Methods Mol Biol 2011;711:203-226. DOI: 10.1007/978-1-61737-992-5_9

24. Schirmer T, Wener B, Hancu I, Martin E. SNR measurements in a single voxel MRS experiment. Proc Intl Soc Mag Reson Med 2005;13:2504.

25. Li BS, Regal J, Gonen O. SNR versus resolution in 3D 1H MRS of the human brain at high magnetic fields. Magn Reson Med 2001;46(6):1049-1053. DOI: 10.1002/mrm.1297

26. Gonen O, Gruber S, Li BS, Mlynárik V, Moser E. Multivoxel 3D proton spectroscopy in the brain at 1.5 versus 3.0 T: signal-to-noise ratio and resolution comparison. Am J Neuroradiol 2001;22(9):1727-1731.

27. Edelstein WA, Glover GH, Hardy CJ, Redington RW. The intrinsic signal-to-noise ratio in NMR imaging. Magn Res Imag 1986;3:604-618. DOI: 10.1002/mrm.1910030413

28. Alecci M, Brivati JA, Placidi G, Testa L, Lurie DJ, Sotgiu A. A submicrosecond resonator and receiver system for Pulsed Magnetic Resonance with large samples. J Magn Reson 1998;132:162-166. DOI: 10.1006/jmre.1998.1398

29. Kreis R. Issues of spectral quality in clinical 1H-magnetic resonance spectroscopy and a gallery of artifacts. NMR Biomed. 2004;17:361-381. DOI:10.1002/nbm.891

30. Giovannetti G. Multiturn surface coil: theoretical considerations on unloaded to loaded Q ratio and SNR. Conc Magn Reson Part B 2014; 44B(2):27-31. DOI: 10.1002/cmr.b.21259 